\title{
Damping and Relative Mode-shape Estimation in Near Real-time through Phasor Approach
}

\author{
Nilanjan Ray Chaudhuri, Member, IEEE, Balarko Chaudhuri, Member, IEEE
}

\begin{abstract}
A technique for estimating damping and electromechanical mode-shape in near real-time as oscillations develop under transient condition is presented. At each sampling instant measured signals are expressed as phasors using corrected values of modal frequencies. Damping is obtained from the exponential variation of estimated phasor magnitude using a moving window Least Squares (LS) algorithm. The relative mode-shape is computed directly from the magnitude and phase angle of the phasors. Random variations in loads are considered to examine possible impact on phasor estimation especially, the frequency correction loop. Accuracy and speed of convergence is validated by comparing the time variation of estimated dampings and relative mode-shapes against the actual values obtained from the linearized models under respective operating conditions. Besides the well-known 4-machine, 2-area test system, a 16-machine, 5area system is considered for illustration of the concept. MonteCarlo simulations are used to capture the statistical variability in estimation as a result of persistent disturbances (e.g.random fluctuations in loads) leading to different signal-to-noise ratios (SNRs). Results from a commercial real-time simulator illustrate the practical feasibility of the proposed approach.
\end{abstract}

Index Terms-Damping, frequency, mode-shape, phasor, Kalman filtering, least squares, Monte-Carlo.

\section{INTRODUCTION}

$\mathbf{S}$ INCE the 1996 blackout in Western Electricity Coordinating Council (WECC) system, there has been renewed emphasis on continuous measurement-based system monitoring to avoid or restrict the spread of such a collapse [1]. Several events of abrupt line-tripping, load and generation shedding took place during this breakup leading to changes in modal behavior of the system [2], [3]. The spread could possibly have been avoided following appropriate operator intervention with accurate and near real-time knowledge of system frequency and damping which are vital indicators of system stress and stability [4], [5]. Relative mode-shapes estimated in near realtime could also provide crucial information about oscillation interaction paths and in turn might become critical for corrective actions like generator and load tripping.

Estimation of damping ratio and frequency from measured signals is widely reported in the literature. Standard techniques for ringdown/transient response are primarily block-processing algorithms like Prony analysis [6], Minimal Realization Algorithm [7], Steiglitz-McBride Algorithm, Eigensystem Realization Algorithm [8], Matrix-Pencil, Hankel Total Least Squares (HTLS) [9] and Hilbert-Huang transformation (HHT)

Support from the EPSRC UK under grant EESC P11121 is gratefully acknowledged.

N. Chaudhuri and B. Chaudhuri are with the Control and Power Research group, Imperial College London, London, UK (e-mail: n.chaudhuri@imperial.ac.uk, b.chaudhuri@imperial.ac.uk).
[10]. Under ambient condition block-processing techniques including spectral analysis [11], Frequency Domain Decomposition (FDD) [3], versions of Yule-Walker (YW) algorithm [12], numerical sub-space system identification (N4SID) technique [13] and recursive techniques like Least Mean Squares (LMS) adaptive filtering [14], Robust Recursive Least Squares (RRLS) [15] and Regularized RRLS (R3LS) [5] are reported. Some of the block processing algorithms mentioned in [4] and recursive techniques like RRLS and R3LS can be applied to ringdown response as well. It has been shown recently that versions of YW [4] and recursive methods like RRLS and R3LS can be applied for near real-time applications.

Although a lot of research attention has been focused on damping and frequency estimation, not many papers exist on mode-shape estimation especially, in near real-time [16]. Identification of mode-shapes in ringdown scenario has been carried out with Matrix Pencil [17], Prony [18], momentmatching [19] and principal component analysis (PCA) [20] techniques. Small-signal continuous modal parameter estimation technique was used in [21]. Spectral correlation analysis [22], [16] and FDD [3] have been proposed for relative modeshape estimation from ambient data. In [23] a communication method and a channel-matching approach were compared against the spectral technique. Improvements were proposed for the channel-matching approach in [24] by eliminating the Narrow Bandpass Filter (NBF) and a similar technique was employed in [25] using a Least-Squares (LS) algorithm based transfer-function (TF) approach. The TF approach was shown to be more accurate compared to the spectral technique [25] for ringdown/transient response.

In $\mathrm{TF}$ approach the transfer-function between two output signals is estimated which in effect is the ratio of the numerators of individual output-input TFs. Accurate estimation of numerator is not always straightforward. Moreover, the estimated TF might be non-causal [25] requiring the knowledge of channel delay - which could be an issue for a near real-time realization. The communication approach [23] on the other hand is non-parametric and seems to be more appropriate. However a Narrow Band Filter (NBF) is required to preprocess the signal as it is suitable for handling only one mode at a time. NBFs need the precise knowledge of center frequency and are typically high-order filters causing delays. For practical applications the modal frequencies can change with operating conditions requiring online adjustment of the center frequency.

In this paper a phasor approach is proposed for estimation of damping and relative mode-shape in near real-time during transient conditions. The proposed technique is in line with 
the communication approach [23] but eliminates its abovementioned drawbacks by using Recursive Kalman Filtering algorithm along with online correction of modal frequencies. The measured signal is decomposed into an average value and a number of oscillatory components expressed as phasors in rotating $\mathrm{d}-\mathrm{q}$ reference frames. The time-varying magnitudes of the phasors capture the oscillation amplitudes at every sampling instant which allows computation of damping ratios through a moving window least squares error (LSE) approach. Relative magnitudes and phase angles of the mode-shape are directly estimated from the variation of amplitudes and phase angles of the phasors with respect to a reference.

The phasor estimation algorithm is initialized with frequencies obtained during ambient condition. These frequencies are corrected online with changes in operating conditions. Moreover, de-trending of the measured signal is automatically achieved in near real-time with time-varying dc offset captured in the average part. Unlike parametric approaches (e.g. TF), which suffer from over-sampling due to extrapolation effects [26], the phasor approach works satisfactorily with any sampling rate above a minimum value.

Case studies on the well-known 4-machine 2-area system and the 16-machine 5-area system demonstrate the effectiveness of the methodology. Monte-Carlo simulations are carried out to investigate the statistical variability in estimations due to random variations in loads. Real-time simulation is also performed to ascertain the practical feasibility due to computational burden.

The contribution of this paper can be summarized as follows:

- A phasor approach is proposed for relative mode-shape estimation in near real-time during transient/ringdown conditions

- The proposed technique is also shown to be very effective for estimation of damping ratio and modal frequency in near real-time

- Extensive simulation studies are carried out on two test systems under different scenarios to prove the effectiveness under transient condition

- The feasibility of the proposed method for near real-time applications is illustrated through real-time simulation

\section{PhASOR APPROACH}

Measured signals during transient condition consists of two components: constant or slowly time-varying average and oscillatory components with one or more modal frequencies. The idea of the phasor approach is to extract the oscillatory component(s) as space-phasor(s) in synchronously rotating dq reference frame(s) [27]. Thus the measured signal $S(t)$ can be expressed as:

$$
S(t)=S_{a v}(t)+\operatorname{Re} \sum_{i=1}^{m}\left\{\vec{S}_{p h(i)} e^{j \omega_{i} t}\right\}
$$

where $m$ is the number of modes in the signal. The space phasor is decomposed into $\mathrm{d}$ and $\mathrm{q}$ components in the individual rotating reference frame as follows:

$$
S(t)=S_{a v}(t)+\sum_{i=1}^{m}\left\{S_{d(i)}(t) \cos \varphi_{i}(t)-S_{q(i)}(t) \sin \varphi_{i}(t)\right\}
$$

Here, $\varphi_{i}(t)=\omega_{i} t+\varphi_{0(i)}$ and $\varphi_{0(i)}$ is the angle at which each estimated space-phasor gets locked with its d-q frame of reference.

Recursive Kalman filter estimation approach is adopted to estimate the parameter vector $\Theta=$ $\left[S_{a v}(t) S_{d(1)}(t) \ldots S_{d(m)}(t) S_{q(1)}(t) \ldots S_{q(m)}(t)\right]^{T} \quad$ following the standard steps [28], [29] shown below:

Step I: Calculate the prediction error:

$$
\varepsilon(t)=S(t)-\phi(t) \Theta(t-1)
$$

where, $\phi(t)$ is the regressor expressed as:

$$
\phi(t)=\left[1 \cos \varphi_{1}(t) \ldots \cos \varphi_{m}(t)-\sin \varphi_{1}(t) \ldots-\sin \varphi_{m}(t)\right]
$$

Step II: Compute the Kalman gain vector $K_{d}(t)$ :

$$
\mathrm{K}_{d}(t)=\frac{\wp(t-1) \phi^{T}(t)}{R_{2}+\phi(t) \wp(t-1) \phi^{T}(t)}
$$

Step III: Update the covariance matrix $\wp(t)$ :

$$
\wp(t)=\left[I-\mathrm{K}_{d}(t) \phi(t)\right] \wp(t-1)+R_{1}
$$

Step IV: Update parameter vector $\Theta(t)$ :

$$
\Theta(t)=\Theta(t-1)+\mathrm{K}_{d}(t) \varepsilon(t)
$$

The parameter vector $\Theta(t)$ is initialized with zeros while the covariance matrix $\wp(t)$ with a high value $\left(10^{4} I\right) . R_{1}$ is a diagonal matrix normalized with respect to $R_{2}$ which results in $R_{2}$ being an unity matrix. Choice of $R_{1}$ depends on the process noise covariance which is difficult to know a-priori. Hence, $R_{1}$ is tuned to attain a proper balance between the filter convergence speed and tranquillity in parameter estimates [28], [29]. Here the elements of $R_{1}$ were chosen to be 0.5 [28].

The basic concept of phasor extraction is illustrated in Fig. 1. The modal frequencies can be initialized with their

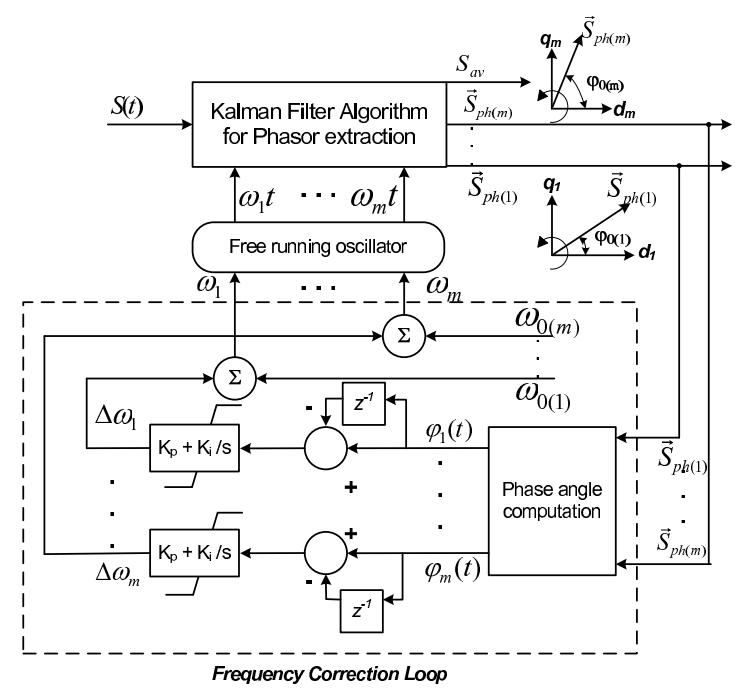

Fig. 1. Extraction of oscillatory components in phasor approach 
nominal values obtained from linear analysis which might not always be straightforward for large practical systems. To avoid this a self-initialization method is employed where the estimation algorithms appropriate for ambient conditions are used to obtain the frequency spectrum of the measured data to initialize the frequencies. It should be mentioned that historical data or operator experience might also play a key role in this process [16]. This initial frequency is then updated with time depending on the actual measurements.

Changes in frequencies with varying operating condition is tracked online through frequency correction loops for individual modes wherein PI compensators minimize the error between the phase angles in consecutive samples, see the dotted box in Fig. 1. The frequency corrections are limited to $\pm 0.1 \mathrm{~Hz}$ to avoid overlap between adjacent frequencies present in a multi-modal signal. If more than one mode with same or very close frequencies exist, the algorithm interprets the signal as a superposition of identical oscillatory modes and fails to identify them individually. This happens as there can be different interpretations of the same signal [30] which could be challenging. A free-running oscillator (e.g. a VCO) can be employed for integrating the frequencies $\omega_{i}$ to generate the angles $\varphi_{i}(t)$.

\section{A. Estimation of Damping and Frequency}

In phasor approach, the oscillatory component of the measured signal is transformed into a set of phasors containing the amplitudes of individual modes. The $i^{t h}$ estimated phasor in time domain can be expressed as:

$$
\left|\vec{S}_{p h(i)}\right|=\sqrt{S_{d(i)}^{2}(t)+S_{q(i)}^{2}(t)}=S_{0(i)} e^{-\frac{\xi}{\sqrt{1-\xi^{2}}} \omega_{i} t}
$$

where, $\xi$ is the damping ratio, $S_{0(i)}$ is the amplitude of the phasor at $t=0 \mathrm{~s}$ and $\omega_{i}$ is the corrected frequency for the $i^{t h}$ mode. Assuming $\chi(t)=\frac{\xi}{\sqrt{1-\xi^{2}}} t$, natural logarithm on both sides of (8) give:

$$
\chi(t)=-\frac{1}{\omega_{i}} \ln \left\{\frac{\left|\vec{S}_{p h(i)}\right|}{S_{0(i)}}\right\}=p_{1} t+p_{0}
$$

As a result, $\chi(t)$ can be expressed as a linear function of time $t$. The coefficients $p_{1}$ and $p_{0}$ can be estimated using a moving window least squares error (LSE) approach as follows:

$$
\begin{gathered}
\mathrm{P}=\left(\Gamma^{T} \Gamma\right)^{-1} \Gamma^{T} \mathrm{X}(t) \\
\mathrm{P}=\left(\begin{array}{c}
\hat{p}_{1} \\
\hat{p}_{0}
\end{array}\right), \Gamma=\left[\begin{array}{ccccc}
0 & T_{s} & \cdot & \cdot & (M-1) T_{s} \\
1 & 1 & . & . & 1
\end{array}\right]^{T}
\end{gathered}
$$

where $\mathrm{X}(t)=\left[\begin{array}{llll}\chi\left(t_{1}\right) & \chi\left(t_{2}\right) & . & \cdot \chi\left(t_{M}\right)\end{array}\right]^{T}$ is the measurement vector.

It is important to note the following points:

1) The most recent sample $\chi(t)$ is included in the window and the oldest one is discarded

2) Closer observation of (8) reveals that a data window at any particular time instant will have the time reference i.e. $t=0 \mathrm{~s}$ corresponding to the first sample in the window. As a result $S_{0(i)}$ is the first sample of $\left|\vec{S}_{p h(i)}\right|$ in the window

3) Following point (2) above, the regression matrix $\Gamma$ becomes a constant (see (11)) and thus the pseudoinverse of $\Gamma$ can be calculated off-line - thereby reducing the computational burden

4) $\omega_{i}$ is updated at each sampling instant by the frequency correction loop

5) $p_{0}$ is included in the formulation of LSE to account for any time-independent component present - normally this will be zero

The damping-ratio can be calculated in terms of the estimated parameters $\mathrm{P}$ as:

$$
\xi=\frac{\hat{p}_{1}}{\sqrt{1+\hat{p}_{1}^{2}}}
$$

\section{B. Estimation of Mode-shape}

Dynamic behavior of power systems around a nominal condition can be expressed in state-space form as:

$$
\Delta \dot{x}=A \Delta x+B \Delta u
$$

Here, $\Delta x$ is the power system state-vector including generator angles, speeds etc. The eigenvalues $\lambda_{i}, i=1,2, \ldots, n$ are the roots of the characteristic equation:

$$
\left|A-\lambda_{i} I\right|=0
$$

The right eigenvector $\phi_{i}$ corresponding to the eigenvalue $\lambda_{i}$ satisfies the following equation:

$$
A_{i} \phi_{i}=\lambda_{i} \phi_{i}
$$

where $\phi_{i}$ is also the mode-shape which is a complex vector representing the participation of state variables in the $i^{\text {th }}$ mode. The mode-shape vector can be normalized with respect to any of the state variables and expressed as relative mode-shape as is done throughout the rest of the paper. For each mode, the relative mode-shape is the normalized magnitude and phaseshift between the state trajectories in time-domain which can be represented as phasors.

For validating signal based mode-shape estimation algorithms, generator speeds/internal angles (state-variables) could be used. Since it is difficult to measure internal angles of generators, speed signals were considered in this work. During phasor extraction and frequency correction the estimated phasor $\vec{S}_{p h(i)}$ changes its position dynamically and finally locks itself at an angle $\varphi_{0(i)}$ with the d-q frame. Relative modeshape can thus be estimated using the following steps:

1) Express the speed of the reference generator in phasor form considering the particular mode of interest

2) Use the output of the frequency correction loop of the reference phasor to estimate the corresponding phasors from other speed signals. This would ensure that the other phasors are locked with respect to the reference

3) Finally, compute the relative phasor magnitudes and angles with respect to the reference phasor

This concept is illustrated in Fig. 2 for the estimation of relative mode-shape of $i^{t h}$ state variable in $j^{t h}$ mode. 


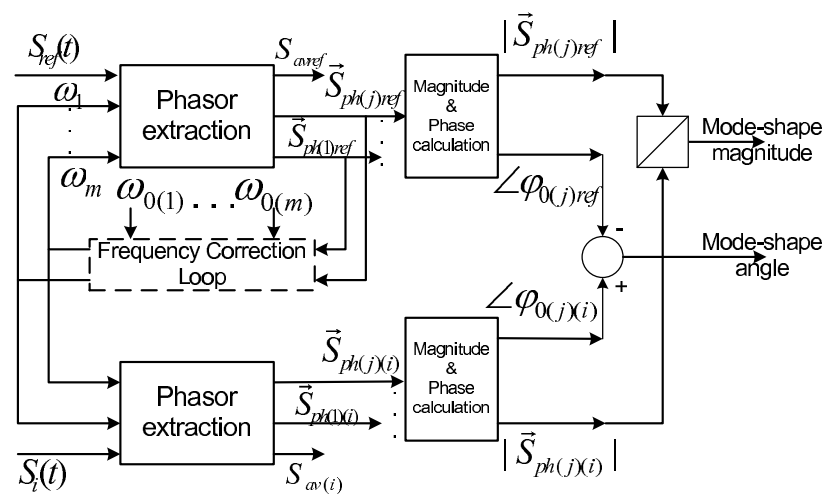

Fig. 2. Estimation of relative mode-shape for the $i^{t h}$ state variable in the $j^{\text {th }}$ mode with phasor approach

\section{Switching Strategy}

Satisfactory performance under both ambient and transient condition is ensured with the switching strategy shown in Fig. 3. As the proposed phasor approach is more effective

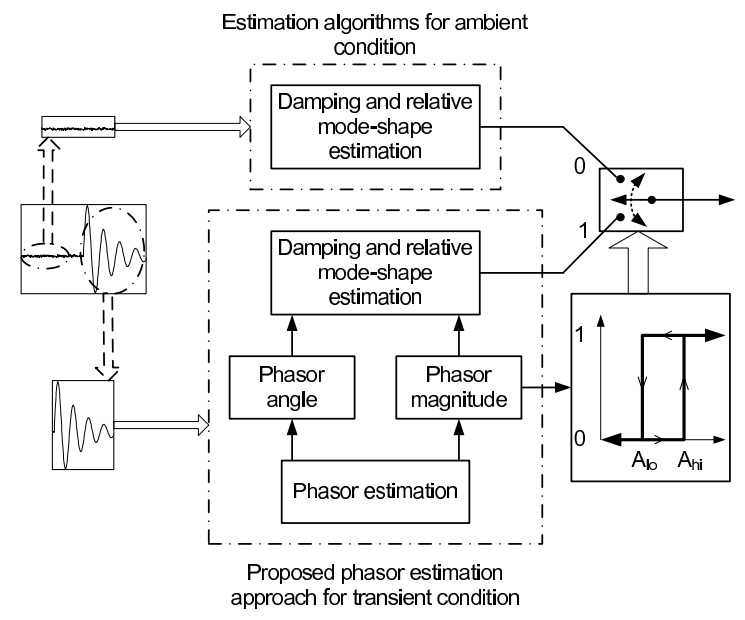

Fig. 3. Switching strategy for the proposed technique using an event detection logic

under ringdown/transient conditions, an event detection logic with an appropriate dead-band is used to switch to the output of the phasor estimator. Transient condition is detected when the phasor magnitude exceeds a threshold $A_{h i}$. Below a certain threshold $A_{l o}$ (see Fig. 3), other algorithms effective for ambient condition [4], [3] are used. Similar logic based arrangement was applied in a prototype Oscillation Monitoring System (OMS) at Tennessee Valley Authority (TVA) [31].

\section{CASe Study I: 4-Machine, 2-ARea System}

The first case study is based on the well known 4-machine, 2-area system shown in Fig. 4 [32]. In steady state, approximately $400 \mathrm{MW}$ flows from area 1 to area 2. To control this tie-line power flow, a thyristor controlled series capacitor (TCSC) is installed to provide $10 \%$ compensation in steady state. Further details of the system can be found in [32]. Integrated Gaussian noise is injected at the load buses to emulate random variations in loads. Monte-Carlo simulation

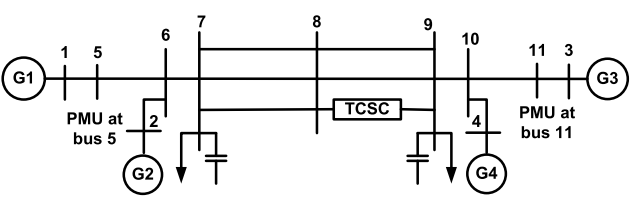

Fig. 4. Test system I: 4-machine, 2-area system with a TCSC

study based on 100 trials was carried out to investigate the statistical variability in estimation.

For all the case studies, estimated $d$ and $q$ components of the phasor as well as the output of frequency correction loop were low-pass filtered to minimize the impact of system noise. The accuracy of the estimates are compared against the actual values obtained from linear analysis. Also a comparison with the dampings and frequencies estimated by Prony analysis is presented in the Appendix. For damping estimation the difference between phase angles of voltages at buses 5 and 11 was considered, whereas mode-shape was estimated using generator speed measurements (typical traces are shown in Fig. 5).
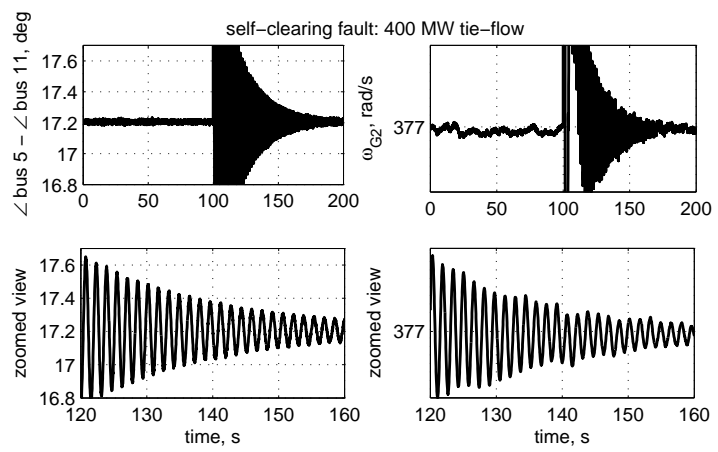

Fig. 5. Upper traces: Typical variation of difference between phase angles of voltage at buses 5 and 11 and speed of generator G2 (see Fig. 4) under ambient and transient conditions. Lower traces: zoomed view of sections of the transient parts

To illustrate the feasibility of the switching strategy described in Section II-C the evolution of estimated phasor magnitude from ambient to transient is shown in Fig. 6. Estimated dampings, frequencies and mode-shapes from phasor algorithm would be used only when the phasor magnitude exceeds a pre-specified threshold indicating onset of transient condition.

\section{A. Estimation of Damping and Frequency}

The modal frequency of the inter-area modes could be initialized with their nominal values obtained from eigen analysis. However, due to the reasons mentioned in Section II, the ambient spectrum of measured phase angle difference between buses 5 and 11, see Fig. 7 was used to obtain the initial guess of the frequency. Frequency correction is initiated once the estimated phasor magnitude exceeds a certain threshold. A $5 \mathrm{~s}$ moving-window was chosen for damping estimation with LSE algorithm.

The estimated damping and frequency are shown in Fig. 8. Following a self-clearing fault for $80 \mathrm{~ms}$ near bus 8 under 

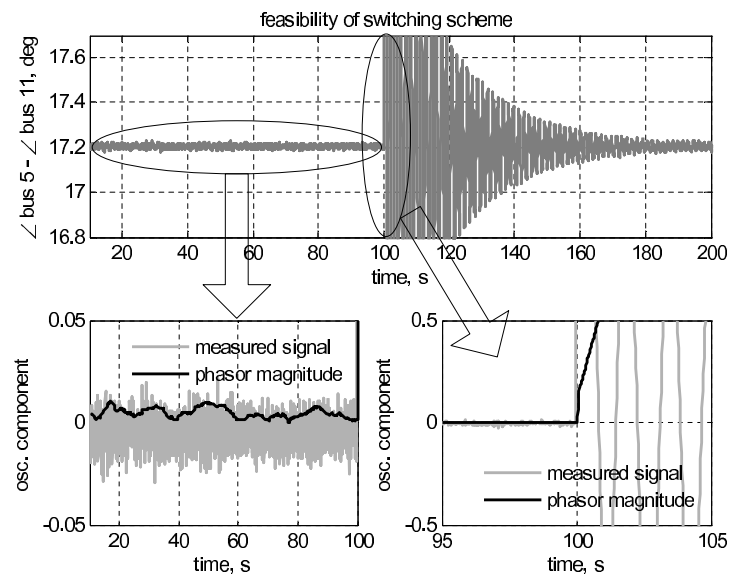

Fig. 6. Upper trace: Typical variation of difference between phase angles of voltage at buses 5 and 11 (see Fig. 4) under ambient and transient conditions. Lower traces: zoomed view of sections of the oscillatory component under ambient and transient along with the estimated phasor magnitude

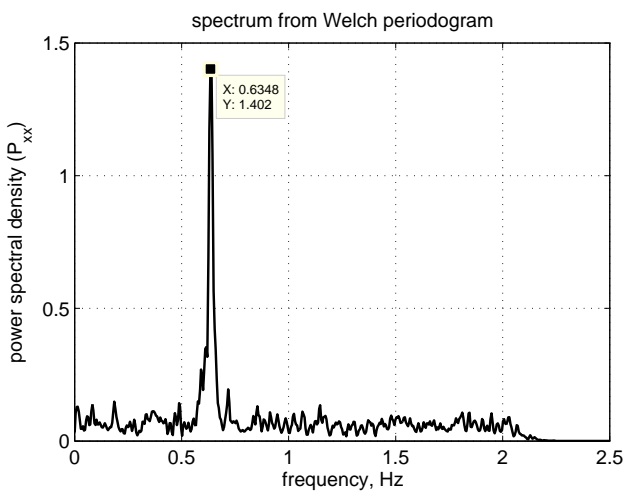

Fig. 7. Ambient spectrum of the measured phase angle difference between buses 5 and 11, see Fig. 4

nominal operating condition (400 MW tie-flow), the estimated frequency settles to the pre-fault value after initial variations due to convergence of Kalman Filter algorithm. The damping estimation also converges to the accurate value within 10-12 $\mathrm{s}$ after the disturbance.

Similar trend is observed under heavy loading condition (825 MW tie-flow) following the same fault, see Fig. 8. As expected, damping of the inter-area mode becomes poorer with heavier loading. Moreover, the frequency of oscillation changes by about $0.07 \mathrm{~Hz}$ from the nominal condition which is reasonably tracked by the frequency correction loop. Results with a three-phase fault near bus 8 followed by line 8-9 outage also illustrates satisfactory convergence, see Fig. 8.

\section{B. Estimation of Mode-shapes}

The compass plot of the relative mode-shapes (obtained from linear analysis) of generator speeds under two operating conditions is shown in Fig. 9. For this case study the speed of generator 3 is taken as reference. Under transient condition the speed signal contains exponentially decaying dc component which is estimated as the average value thus achieving effective de-trending in near real-time. In Figs. 10 and 11 the estimated relative mode-shapes are plotted.

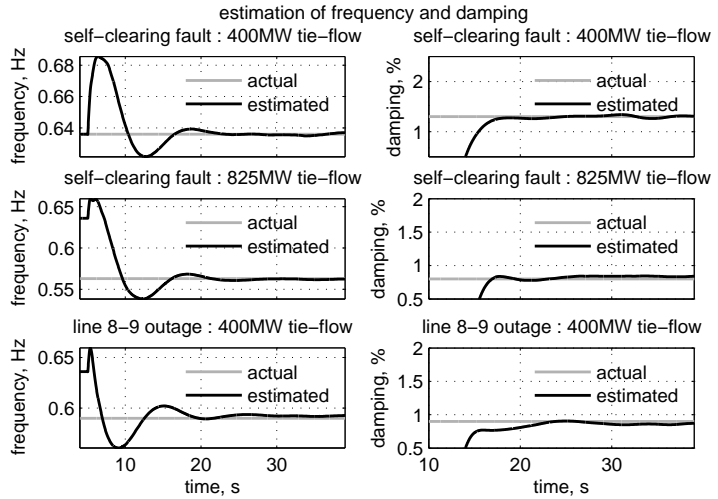

Fig. 8. Estimated damping-ratio and frequency across different operating conditions: a three-phase fault of 5-cycle duration occurs at $t=5 \mathrm{~s}$.

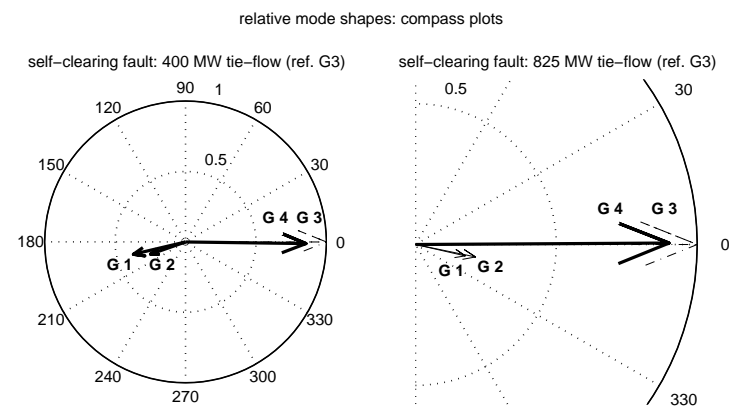

Fig. 9. Compass plot of relative mode-shapes obtained from linear analysis

Consecutive disturbances were simulated to illustrate the speed and accuracy of convergence. A representative result considering a three-phase fault near bus 8 cleared by line 7-8 outage at $t=40 \mathrm{~s}$ and subsequent line reclosure at $t=80 \mathrm{~s}$ is presented here. Fig. 10 shows that the changes in system damping and relative mode-shape from one operating condition to the other is tracked with reasonable speed and accuracy. This figure also shows continuous frequency tracking under different operating scenarios starting with the nominal value.

Under heavy loading condition with 825 MW tie-flow, mode-shape of the system changes significantly from nominal condition (as estimated during line reclosure in Fig. 10) where all the generators swing nearly in phase [32], see Fig. 9. In Fig. 11 little variations in estimates are observed around the actual values perhaps due to higher system non-linearities under heavy loading.

Fig. 12 shows the mean and standard deviation of estimated damping ratio and mode-shape magnitudes obtained from 100 Monte-Carlo trials. Due to space restrictions, statistical variability of relative mode-shape is presented only for one operating condition. As oscillations settle, ambient condition with higher influence of noise is approached moving away from the ringdown situation - hence the standard deviation is increased. It is to be noted that the onset of ambient condition will be reflected in estimated phasor magnitude based on which an algorithm suited for ambient condition will take over from the phasor algorithm following the switching scheme in 

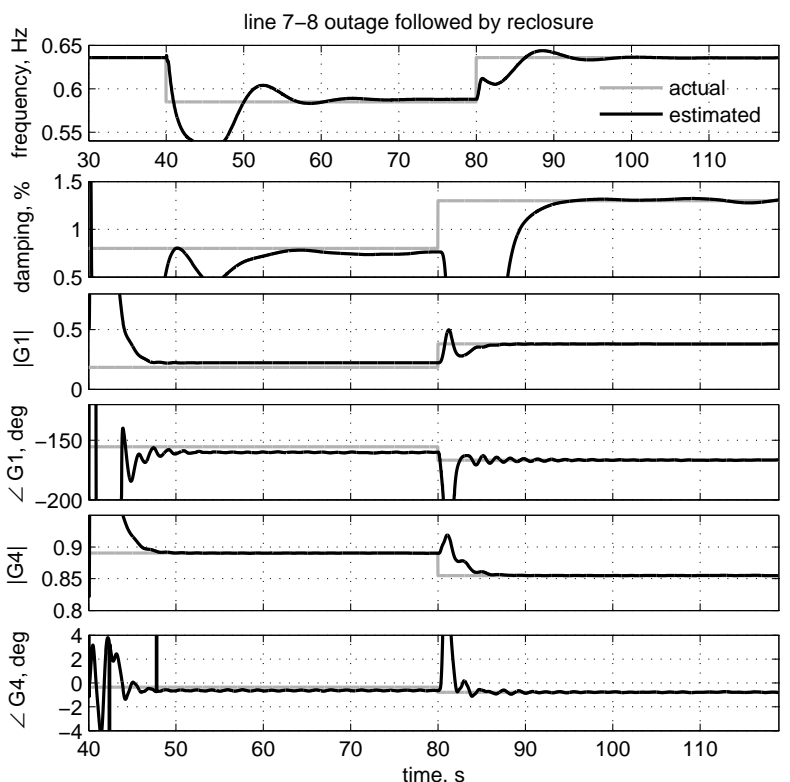

Fig. 10. Estimated damping and relative mode-shapes considering line 78 outage at $\mathrm{t}=40 \mathrm{~s}$ after a three-phase fault near bus 8 followed by line reclosure at $t=80 \mathrm{~s}$. Reference generator: G3.
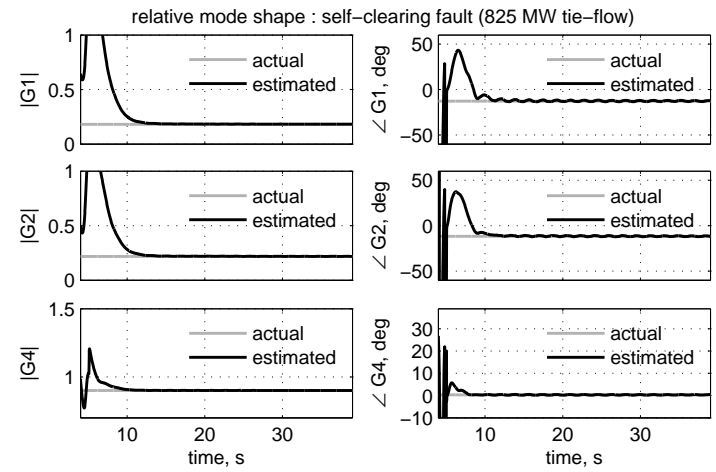

Fig. 11. Estimated relative mode-shapes after a self-clearing fault near bus 8 at $t=5 \mathrm{~s}$ in $825 \mathrm{MW}$ tie-flow condition. Reference generator: G3.

Fig. 3.

To illustrate the effect of signal to noise ratio (SNR) on the estimation, Monte-Carlo simulations with different values of SNR were done. In Fig. 13 the boxes show the statistical spread (median, lower and upper quartile) of the estimated damping, frequency and relative mode-shape in comparison with their actual values (dotted line). For obvious reason the estimation is more consistent across different Monte-Carlo trials for higher values of SNR. However, in all cases the estimated quantities are quite close to their actual values indicating the effectiveness of the proposed algorithm. Modeshape magnitude and phase angle of only one generator, $G 4$ is shown here due to space restrictions.

\section{CASe Study II: 16-Machine, 5-AREA System}

Following basic understanding with the 4-machine 2-area system, a 16-machine, 5-area test system, shown in Fig. 14 was considered. A detailed description of the study system

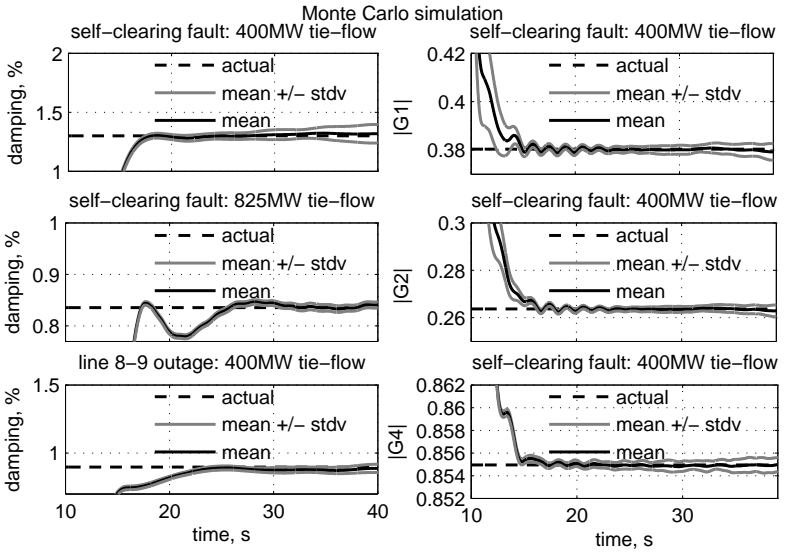

Fig. 12. Monte-Carlo simulation results for estimation of damping and relative mode-shape magnitudes.

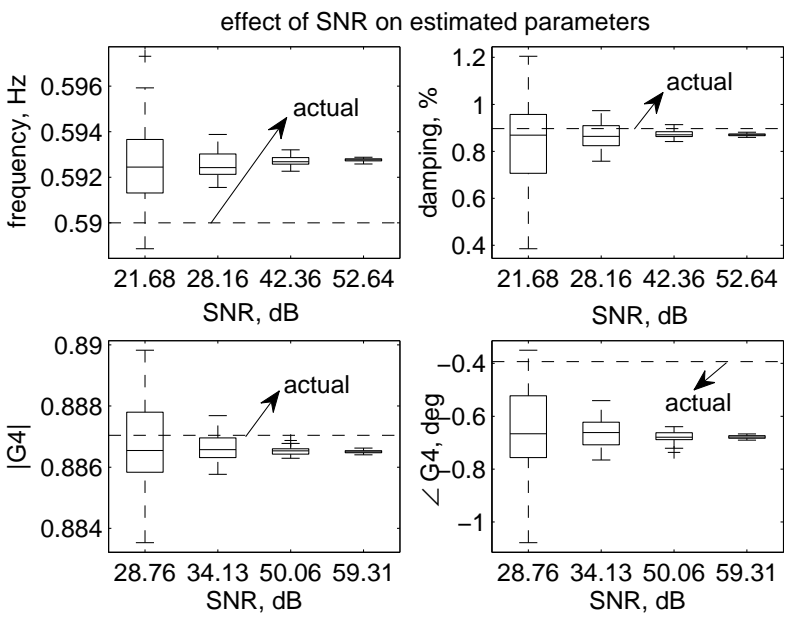

Fig. 13. Effect of SNR on estimated damping, frequency and relative modeshape after a three-phase fault at $t=5 \mathrm{~s}$ near bus 8 followed by line 8-9 outage. Boxplots show the lower quartile, median, upper quartile and extent of the rest of the data and any outliers.

including machine, excitation system and network parameters can be found in [33]. A TCSC is installed on the tie-line connecting buses 18 and 50 . There are three critical inter-area modes with nominal frequencies: $0.398 \mathrm{~Hz}$ (mode \# 1), 0.525 $\mathrm{Hz}$ (mode \# 2) and $0.623 \mathrm{~Hz}$ (mode \# 3).

Integrated Gaussian noise is injected at all load buses to simulate random load fluctuations. Similar to the previous case study, Monte-Carlo simulation based on 100 trials was carried out to investigate the statistical variations. Although both mean and standard deviations of the estimated parameters were monitored for all scenarios, only the results for a particular trial are shown here for clarity. Phase angle difference between voltages at buses 45 and 51 was used for damping estimation, whereas generator speeds were considered for mode-shape estimation (typical traces are shown in Fig. 15).

\section{A. Estimation of Damping and Frequency}

The ambient spectrum of the real power flow from bus 16 to 18 , see Fig. 16 was used to obtain the initial guess of 


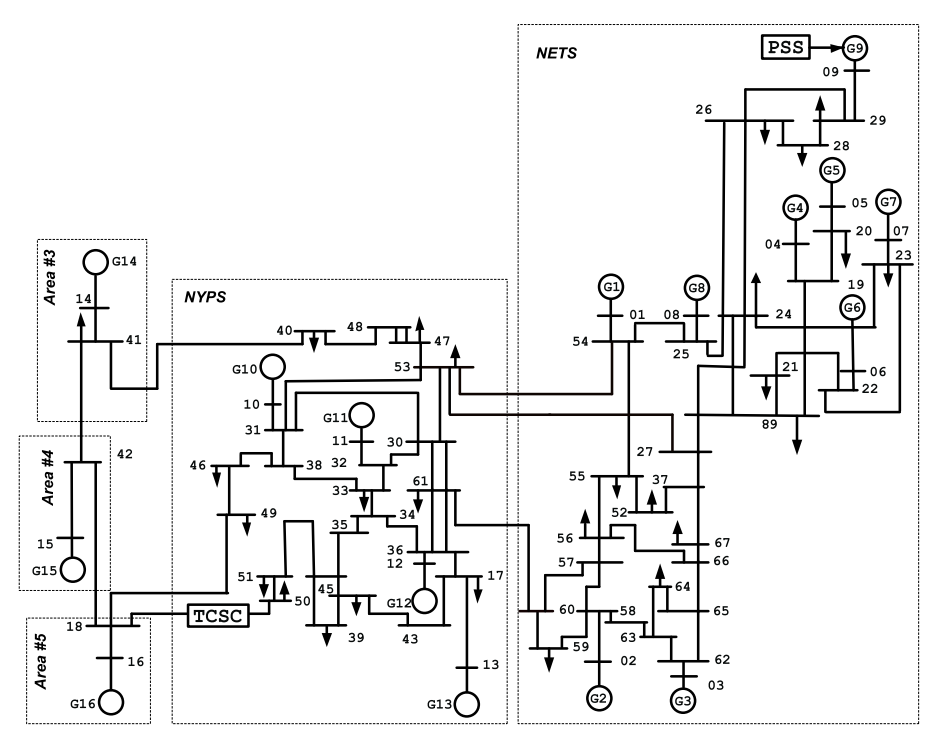

Fig. 14. Test system II: 16-machine, 5-area system with a TCSC.

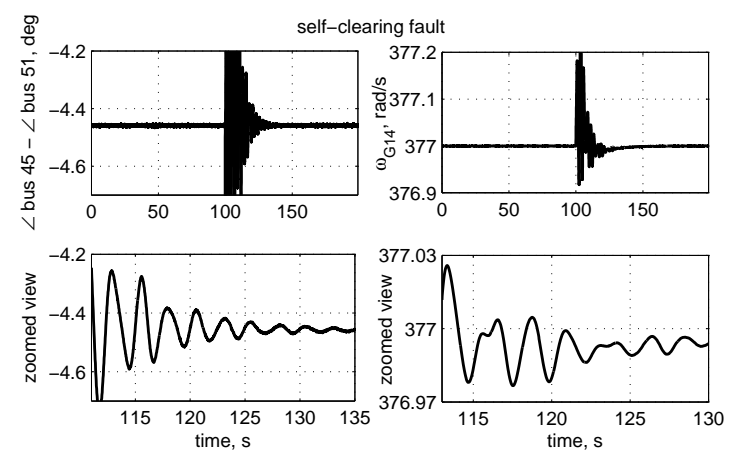

Fig. 15. Upper traces: Typical variation of difference between voltage angles at bus 45 and 51 and speed of generator G14. Lower traces: zoomed view of sections of the transient parts

the frequencies. The performance of the algorithm was tested exhaustively across a wide range of scenarios. Only a few representative cases, as shown in Fig. 17, are reported here due to space restriction. Besides a self-clearing fault near bus 60 , three critical tie-line outages (lines $60-61,54-53$ and 18 42) following three-phase faults at $t=5 \mathrm{~s}$ near one of the respective terminal buses are presented.

Damping estimation for mode \#1 takes about 10-12 s to converge following the first three disturbances. For line 1842 outage, estimation of damping of mode \#2 is illustrated as it is the most critical one - note the performance of the corresponding frequency correction loop in Fig. 17.

\section{B. Estimation of Mode-shapes}

Out of several scenarios considered, only two representative cases following a self-clearing fault near bus 60 and line 54-53 outage are presented here. Compass plots of the relative modeshapes under these conditions obtained from linear analysis are shown in Fig. 18.

Fig. 19 shows the variation of estimated relative modeshapes for mode \# 1 considering one generator from each of the five areas. Angular speed of generator \# 5 is considered as

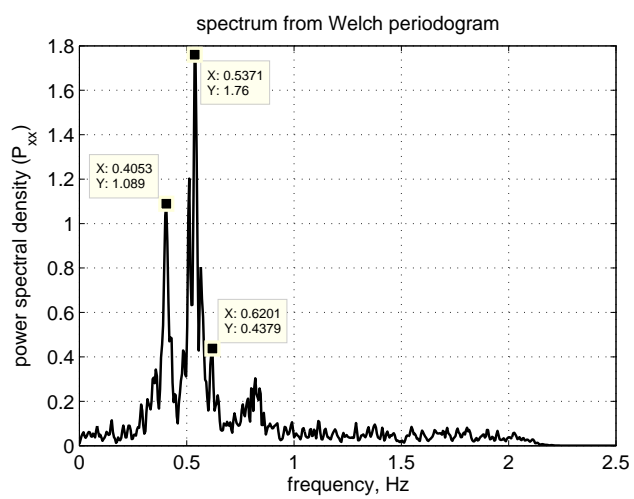

Fig. 16. Ambient spectrum of the measured real power flow between buses 16 and 18 , see Fig. 14

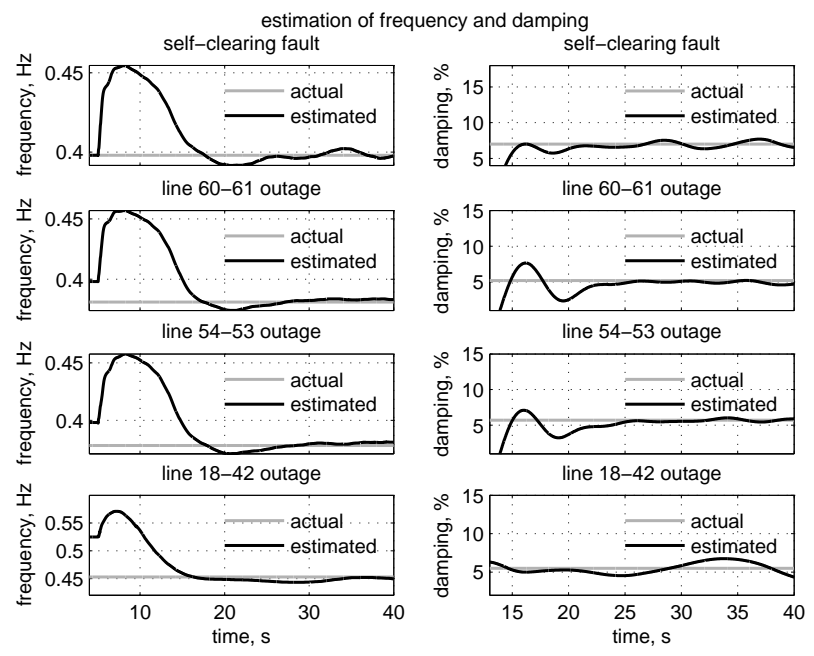

Fig. 17. Estimated damping and frequency across different operating conditions: transient begins at $t=5 \mathrm{~s}$.

the reference for this mode. The relative mode-shape estimates converge to acceptable accuracy within 10-12 s following the disturbance. The estimated mode-shapes reveal that the generators in NETS and NYPS (see Fig. 14) oscillates against those in area \# 3, \# 4 and \#5 which is consistent with Fig. 18. As shown in Fig. 20, estimated mode-shapes following the outage of a tie-line connecting NETS and NYPS (see Fig. 14) also matches the swing patterns obtained from linear analysis, see Fig. 18. Similar trends of convergence were observed for other outage scenarios which could not be presented here due to space restrictions.

A comparison between the two case studies in Sections III and IV shows that in general, the convergence is better for the case study I. For case study II, there are more variations in estimated damping and relative mode-shape about their actual values. This is due to the fact that the test system used for case study II has multiple inter-area modes resulting in multi-modal signals unlike the test system for case study I which has only one inter-area mode. Estimating modal behavior from multimodal signals is challenging especially, when the frequencies are very close to each other. Proper choice of signals with one dominant modal content helps in such situations [16].

Results of Monte-Carlo simulations with different values of 


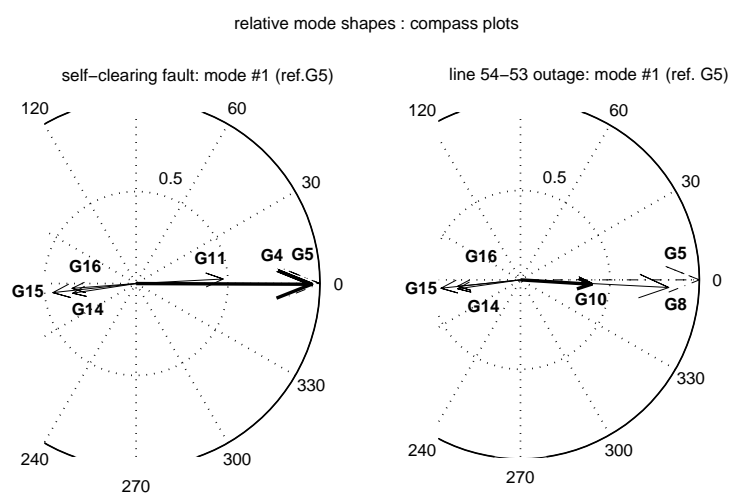

Fig. 18. Compass plot of relative mode-shapes obtained from linear analysis
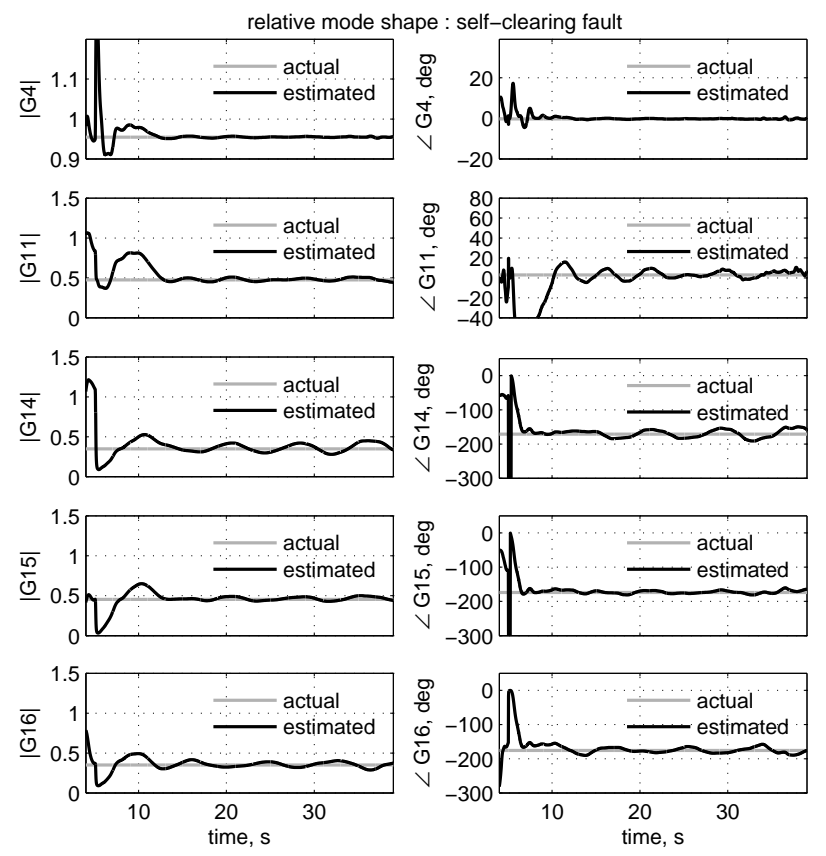

Fig. 19. Estimated relative mode-shapes after a self-clearing fault near bus 60 at $t=5 \mathrm{~s}$. Reference generator: G5.

SNR were are shown in the form of boxplots in Fig. 21. The statistical spread in estimated damping, frequency and relative mode-shape is shown in terms of the median, lower and upper quartiles) with their actual values (dotted line) in the backdrop. Estimation is more consistent across different Monte-Carlo trials for higher values of SNR. However, in all cases the estimated quantities are quite close the respective actual values indicating the effectiveness of the proposed algorithm. Modeshape magnitude and phase angle of only one generator $G 8$, is shown due to space restrictions.

\section{Real-time Simulation}

Damping and relative mode-shape estimation algorithm involve significant mathematical computation (see Section II), within each sampling interval of $20 \mathrm{~ms}$. Real-time simulations were carried out to detect possible overruns in computation and confirm practical feasibility of the proposed approach.
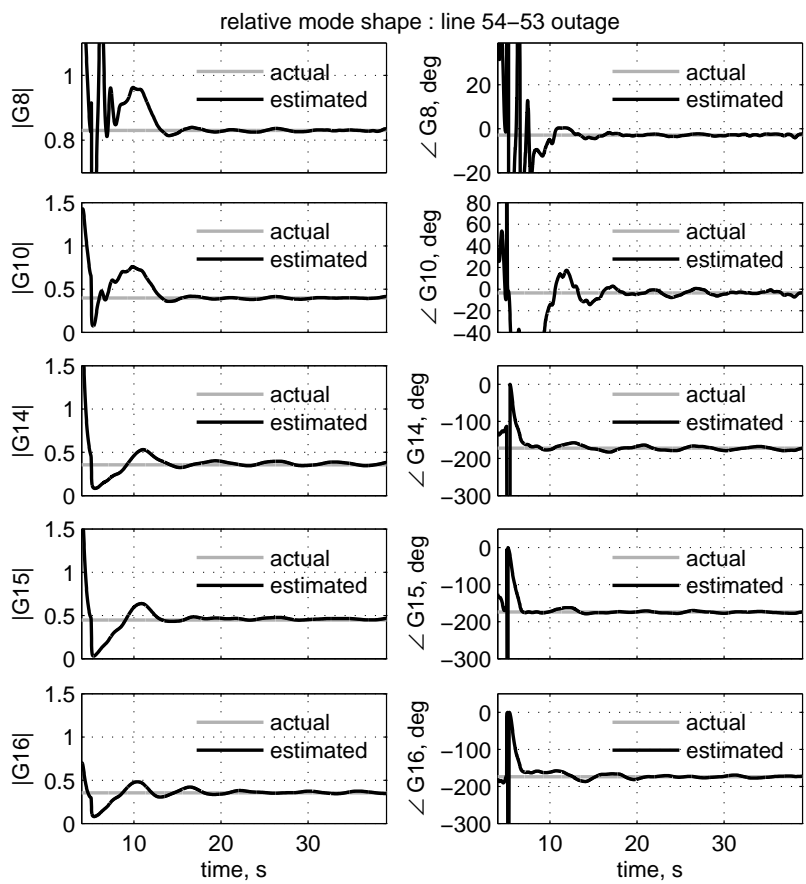

Fig. 20. Estimated relative mode-shapes for mode \# 1 after a three-phase fault near bus 54 followed by line 54-53 outage at $t=5 \mathrm{~s}$. Reference generator: G5.
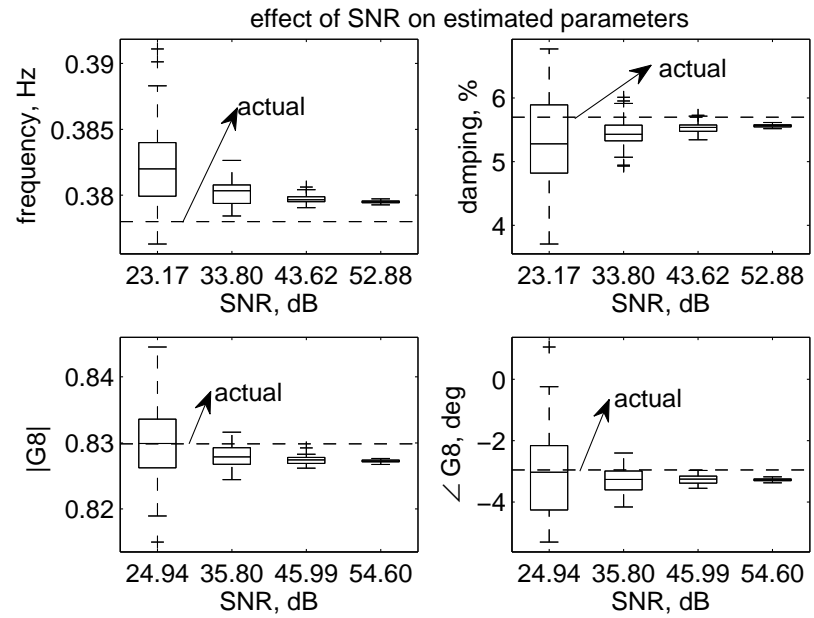

Fig. 21. Effect of SNR on estimated damping, frequency and relative modeshape for mode \# 1 after a three-phase fault at $t=5 \mathrm{~s}$ near bus 54 followed by line 54-53 outage. Boxplots show the lower quartile, median, upper quartile and extent of the rest of the data and any outliers.

The dynamic behavior of the test system shown in Fig. 14 was emulated using an Opal-RT real-time simulator [34]. Analog signals out of the real-time simulator were captured in an oscilloscope and passed on to an oscillation monitor platform for estimation of damping and relative mode-shape in near real-time. Fig. 22 shows the damping and relative modeshape estimation following line 54-53 outage. The oscilloscope traces of the measured signals are shown here for the phase angle difference between buses 45 and 51 along with generator speed deviations of generator \# 5, \# 8 and \# 15. Very close similarity with the computer simulation results, see Figs. 17 and 20 , confirm that the computations could be successfully 


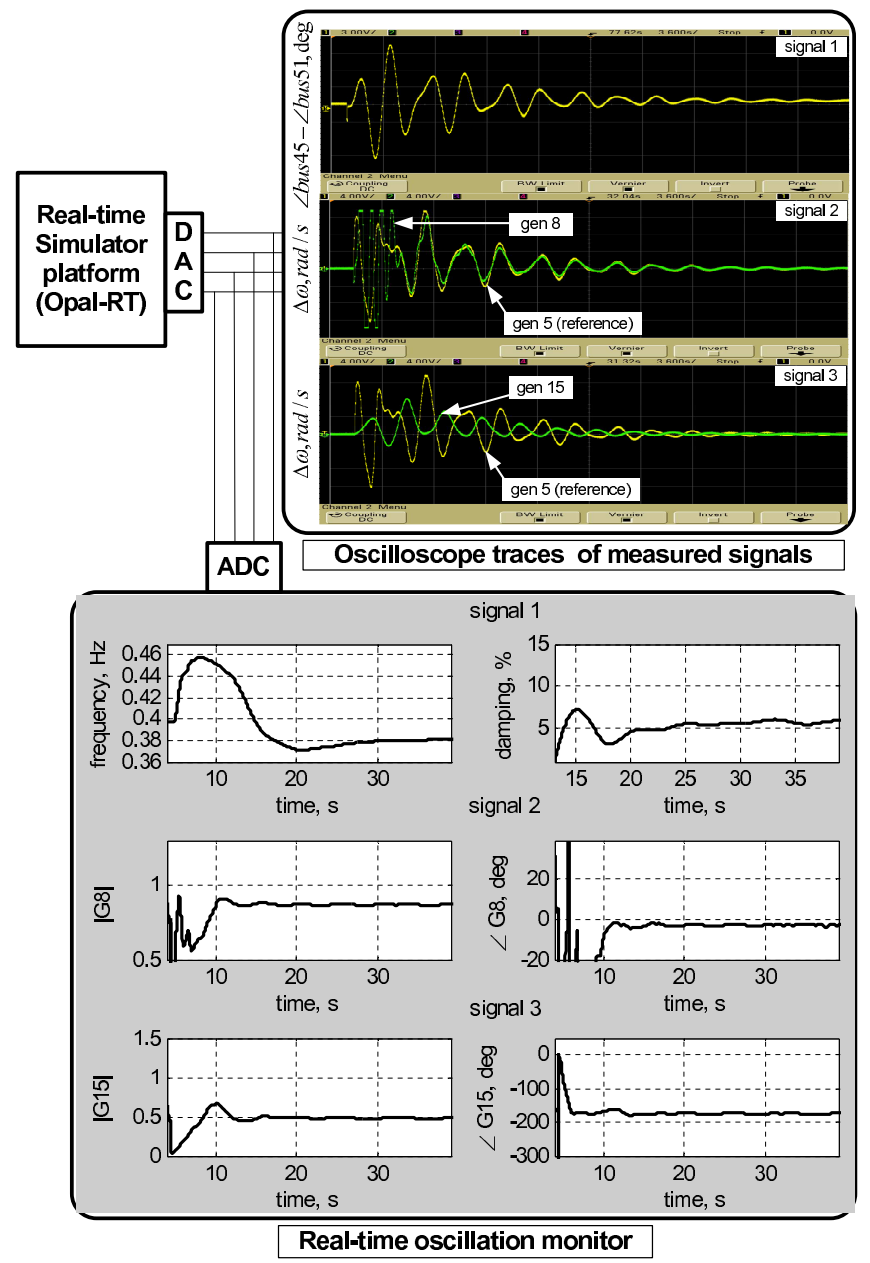

Fig. 22. Oscilloscope traces of measured signals and corresponding damping and mode-shape estimation with phasor approach for line 54-53 outage.

completed within each sampling interval. Not a single instance of overrun was detected in the real-time simulator.

The oscilloscope trace in the middle (see Fig. 22) shows that the speed deviation of generator \#8 initially contains higher frequencies due to local mode, which is clipped by $+/-13.5$ $\mathrm{V}$ limits of the DAC. Hence, there is noticeable difference between the estimated mode-shape of this generator for the real-time study and the simulation study during the timespan of $5-10$ s, compare Figs. 20 and 22. However, the patterns match closely once the initial transients (period of DAC limit violations) are over. No such problem is there for speed deviation of generator \#15 resulting in very similar computer and real-time simulation results.

Although the presence of multiple modes is visible from the traces of generator speeds, mode \#1 is dominant and oscillation pattern of generator \#8 speed deviation shows that it is similar in magnitude and almost in phase with the reference generator. On the other hand, bottom signal shows that oscillation amplitude of the speed of generator \#15 is much lesser and almost out of phase with respect to the reference generator. The same information is reflected in the estimated relative mode-shape magnitudes and phase angles, see Fig. 22. The proposed phasor approach is general and applicable to system of any size. Rather than the size of the system itself, the number of critical modes would influence the computational burden and could be a limiting factor.

\section{CONCLUSION}

A phasor approach based on Recursive Kalman Filtering is presented in this paper for estimating the damping and relative mode-shape in near real-time under transient condition. At each sampling instant measured signals are expressed as phasors using corrected values of modal frequencies. Damping is obtained from the estimated phasor magnitude using a moving window LS curve-fitting approach while the magnitude and phase angle of the phasors directly provide the relative modeshape. Besides the well-known 4-machine, 2-area test system, a 16-machine, 5-area system is considered to demonstrate the methodology. Simulation results across a wide range of operating conditions show that the relative mode-shapes are estimated with acceptable accuracy in transient conditions. Monte-Carlo simulations show that the statistical variability in estimation as a result of random load variations and different signal-to-noise ratios (SNRs) is within acceptable limits. Results from a real-time simulator confirms the practical feasibility of the approach.

\section{ACKNOWLEDGMENT}

The authors would like to thank Prof. John Pierre and Prof. Dan Trudnowski for providing valuable insight into this subject during discussions at the 2009 PES General Meeting in Calgary.

\section{APPENDIX}

\section{A. Comparison With Prony Analysis}

Prony analysis is a block-processing algorithm and has mostly been used for off-line analysis [6] whereas the proposed method is a recursive suitable for a near real-time application. A comparison of the damping and frequency estimated from Prony analysis (with a $30 \mathrm{~s}$ time window) against the linear analysis for the case studies in Section III and IV is shown below:

TABLE I

Comparison against PRony anAlysis: CASE STUdy I

\begin{tabular}{|c||c|c|c||c|}
\hline \multicolumn{1}{|c||}{} & \multicolumn{2}{c|}{ damping, \% } & \multicolumn{2}{c|}{ frequency, $\mathrm{Hz}$} \\
\hline \hline Scenario & Prony & Linear & Prony & Linear \\
\hline \hline 400 MW & 1.28 & 1.3 & 0.636 & 0.636 \\
\hline 8-9 out & 0.92 & 0.90 & 0.5928 & 0.590 \\
\hline 7-8 out & 0.77 & 0.80 & 0.588 & 0.585 \\
\hline 825 MW & 0.83 & 0.84 & 0.562 & 0.563 \\
\hline
\end{tabular}

TABLE II

Comparison against Prony analysis: CASE Study II

\begin{tabular}{|c||c|c||c|c|}
\hline \multicolumn{1}{|c||}{} & \multicolumn{2}{c|}{ damping, \% } & \multicolumn{2}{c|}{ frequency, $\mathrm{Hz}$} \\
\hline \hline Scenario & Prony & Linear & Prony & Linear \\
\hline \hline nominal & 6.83 & 7.0 & 0.397 & 0.398 \\
\hline $60-61$ out & 5.08 & 5.20 & 0.383 & 0.381 \\
\hline $54-53$ out & 5.57 & 5.70 & 0.379 & 0.378 \\
\hline $18-42$ out & 5.47 & 5.50 & 0.447 & 0.453 \\
\hline
\end{tabular}




\section{REFERENCES}

[1] J. Hauer, D. Trudnowski, G. Rogers, B. Mittelstadt, W. Litzenberger, and J. Johnson, "Keeping an eye on power system dynamics," IEEE Computer Applications in Power, vol. 10, no. 4, pp. 50-54, 1997.

[2] A. J. F. Hauer, D. J. Trudnowski, and J. G. DeSteese, "A perspective on wams analysis tools for tracking of oscillatory dynamics," in Proceedings of IEEE Power Engineering Society General Meeting, 2007., 2007. pp. $1-10$.

[3] G. Liu and V. Venkatasubramanian, "Oscillation monitoring from ambient pmu measurements by frequency domain decomposition," in Proceedings of IEEE International Symposium on Circuits and Systems, 2008 (ISCAS 2008), 2008, pp. 2821-2824.

[4] D. J. Trudnowski, J. W. Pierre, N. Zhou, J. F. Hauer, and M. Parashar, "Performance of three mode-meter block-processing algorithms for automated dynamic stability assessment," IEEE Transactions on Power Systems, vol. 23, no. 2, pp. 680-690, 2008.

[5] N. Zhou, D. J. Trudnowski, J. W. Pierre, and W. A. Mittelstadt, "Electromechanical mode online estimation using regularized robust rls methods," IEEE Transactions on Power Systems, vol. 23, no. 4, pp. 1670-1680, 2008.

[6] J. F. Hauer, C. J. Demeure, and L. L. Scharf, "Initial results in prony analysis of power system response signals," IEEE Transactions on Power Systems, vol. 5, no. 1, pp. 80-89, 1990.

[7] I. Kamwa, R. Grondin, E. J. Dickinson, and S. Fortin, "A minimal realization approach to reduced-order modelling and modal analysis for power system response signals," IEEE Transactions on Power Systems, vol. 8, no. 3, pp. 1020-1029, 1993 .

[8] J. J. Sanchez-Gasca and J. H. Chow, "Performance comparison of three identification methods for the analysis of electromechanical oscillations," IEEE Transactions on Power Systems, vol. 14, no. 3, pp. 995-1002, 1999.

[9] G. Liu, J. Quintero, and V. Venkatasubramanian, "Oscillation monitoring system based on wide area synchrophasors in power systems," in Proceedings of Bulk Power System Dynamics and Control - VII. Revitalizing Operational Reliability, 2007 iREP Symposium, 2007, pp. 1-13.

[10] A. R. Messina and V. Vittal, "Nonlinear, non-stationary analysis of interarea oscillations via hilbert spectral analysis," IEEE Transactions on Power Systems, vol. 21, no. 3, pp. 1234-1241, 2006.

[11] J. F. Hauer and R. L. Cresap, "Measurement and modeling of pacific ac intertie response to random load switching," IEEE Transactions on Power Apparatus and Systems, vol. PAS-100, no. 1, pp. 353-359, 1981.

[12] R. W. Wies, J. W. Pierre, and D. J. Trudnowski, "Use of arma block processing for estimating stationary low-frequency electromechanical modes of power systems," IEEE Power Engineering Review, vol. 22 , no. 11, pp. 57-57, 2002.

[13] I. Kamwa, G. Trudel, and L. Gerin-Lajoie, "Low-order black-box models for control system design in large power systems," IEEE Transactions on Power Systems, vol. 11, no. 1, pp. 303-311, 1996.

[14] R. W. Wies, J. W. Pierre, and D. J. Trudnowski, "Use of least mean squares (lms) adaptive filtering technique for estimating low-frequency electromechanical modes in power systems," in IEEE Power Engineering Society General Meeting, 2004, 2004, pp. 1863-1870 Vol.2.

[15] N. Zhou, J. W. Pierre, D. J. Trudnowski, and R. T. Guttromson, "Robust rls methods for online estimation of power system electromechanical modes," IEEE Transactions on Power Systems, vol. 22, no. 3, pp. 12401249, 2007.

[16] D. J. Trudnowski, "Estimating electromechanical mode shape from synchrophasor measurements," IEEE Transactions on Power Systems, vol. 23, no. 3, pp. 1188-1195, 2008.

[17] W. Li, R. M. Gardner, J. Dong, W. Lei, X. Tao, Z. Yingchen, Y. Liu, Z. Guorui, and Y. Xue, "Wide area synchronized measurements and inter-area oscillation study," in Proceedings of IEEE/PES Power Systems Conference and Exposition, 2009. PSCE '09., 2009, pp. 1-8.

[18] R. J. Koessler, F. S. Prabhakara, and A. H. Al-Mubarak, "Analysis of oscillations with eigenanalysis and prony techniques," in IEEE Power Engineering Society General Meeting, 2007, 2007, pp. 1-8.

[19] F. D. Freitas, N. Martins, and L. Fernandes, "Reliable mode-shapes for major system modes extracted from concentrated wams measurements processed by a simo identification algorithm," in IEEE Power and Energy Society General Meeting - Conversion and Delivery of Electrical Energy in the 21st Century, 2008, 2008, pp. 1-8.

[20] K. Anaparthi, B. Chaudhuri, N. Thornhill, and B. Pal, "Coherency identification in power systems through principal component analysis," IEEE Transactions on Power Systems, vol. 20, no. 3, pp. 1658-1660, 2005.
[21] D. H. Wilson, K. Hay, and G. J. Rogers, "Dynamic model verification using a continuous modal parameter estimator," in IEEE Power Tech Conference Proceedings, 2003 Bologna, vol. 2, 2003, p. 6 pp. Vol.2.

[22] M. Banejad and G. Ledwich, "Correlation based mode shape determination of a power system," in Proceedings of IEEE International Conference on Acoustics, Speech, and Signal Processing, 2002 (ICASSP '02)., vol. 4, 2002, pp. IV-3832-IV-3835 vol.4.

[23] L. Dosiek, D. J. Trudnowski, and J. W. Pierre, "New algorithms for mode shape estimation using measured data," in IEEE Power and Energy Society General Meeting - Conversion and Delivery of Electrical Energy in the 21st Century, 2008, pp. 1-8.

[24] L. Dosiek, J. W. Pierre, D. J. Trudnowski, and N. Zhou, "A channel matching approach for estimating electromechanical mode shape and coherence," in IEEE Power and Energy Society General Meeting, 2009, pp. $1-8$.

[25] N. Zhou, Z. Huang, L. Dosiek, D. Trudnowski, and J. W. Pierre, "Electromechanical mode shape estimation based on transfer function identification using pmu measurements," in IEEE Power and Energy Society General Meeting, 2009, pp. 1-7.

[26] K. Worden, "On the over-sampling of data for system identification," Mechanical Systems and Signal Processing, vol. 9, no. 3, pp. 287-297, 1995, doi: DOI: $10.1006 / \mathrm{mssp} .1995 .0024$

[27] L. Angquist and C. Gama, "Damping algorithm based on phasor estimation," in IEEE Power Engineering Society Winter Meeting, vol. 3, 2001, pp. $1160-1165$.

[28] P. E. Wellstead and M. B. Zarrop, Self-tuning Systems. Control and Signal Processing. John Wiley \& Sons, 1991.

[29] M. Zima, M. Larsson, P. Korba, C. Rehtanz, and G. Andersson, "Design aspects for wide-area monitoring and control systems," Proceedings of the IEEE, vol. 93, no. 5, pp. 980-996, 2005.

[30] R. Deering and J. F. Kaiser, "The use of a masking signal to improve empirical mode decomposition," in Proceedings of IEEE International Conference on Acoustics, Speech, and Signal Processing, 2005(ICASSP '05), vol. 4, 2005, pp. iv/485-iv/488 Vol. 4.

[31] G. Liu, V. M. Venkatasubramanian, and J. R. Carroll, "Oscillation monitoring system using synchrophasors," in Power \& Energy Society General Meeting, 2009. PES '09. IEEE, 2009, pp. 1-4.

[32] M. Klein, G. Rogers, and P. Kundur, "A fundamental study of inter-area oscillations in power systems," IEEE Transactions on Power Systems, vol. 6, no. 3, pp. 914-921, 1991.

[33] B. Pal and B. Chaudhuri, Robust control in power systems, ser. Power electronics and power systems. New York: Springer, 2005.

[34] R. Majumder, B. Chaudhuri, B. C. Pal, and C. Dufour, "Real time dynamic simulator for power system control applications," in IEEE Power Engineering Society General Meeting, 2006, p. 7.

Nilanjan Ray Chaudhuri (S'08-M'09) received B.E. degree from Jalpaiguri Government Engineering College, in 2003 and M.Tech. degree from Indian Institute of Technology (IIT), Delhi, in 2005. He was with GE, John F. Welch Technology Center, Bangalore, India, till 2007. He was a research scholar at the University of Western Ontario (UWO), London, ON, Canada. Currently, he is working towards his $\mathrm{PhD}$ at Imperial College London, London, UK. His research interests include power system dynamics and control, application of power electronics in power systems, online system identification, and renewable energy systems.

Balarko Chaudhuri (S'02-M'06) received his $\mathrm{PhD}$ from Imperial College London in 2005. He worked with General Electric Global Research for a year before coming back to Imperial College as a post-doctoral research associate in 2006. He is presently a lecturer in the department of Electrical and Electronic Engineering at Imperial College London. His research interests are in the areas of power system dynamics and stability, FACTS/HVDC, robust control and renewable energy. 